\title{
Atomic force microscopy imaging of the G-banding process of chromosomes
}

Bowei Wang ${ }^{1,2}$, Jiani $\mathrm{Li}^{1,2}$, Jianjun Dong ${ }^{1,2}$, Fan Yang ${ }^{1,2}$, Kaige Qu ${ }^{1,2}$, Ying Wang ${ }^{1,2}$, Jingran Zhang ${ }^{1,2}$, Zhengxun Song ${ }^{1,2}$, Hongmei $\mathrm{Xu}^{1,2}$, Zuobin Wang ${ }^{1,2,4^{*}}$, Huimiao $\mathrm{Wei}^{3 *}$

${ }^{1}$ International Research Centre for Nano Handling and Manufacturing of China, Changchun University of Science and Technology, Changchun 130022, China

${ }^{2}$ Ministry of Education Key Laboratory for Cross-Scale Micro and Nano

Manufacturing, Changchun University of Science and Technology, Changchun 130022, China

${ }^{3}$ Changli Nano Biotechnology Ltd, Changchun 130022, China

${ }^{4}$ JR3CN \& IRAC, University of Bedfordshire, Luton LU1 3JU, UK

*Corresponding author: wangz@,cust.edu.cn; weihuimiao@,163.com

\begin{abstract}
The chromosome is an important genetic material carrier in living individuals and the spatial conformation (mainly referring to the chromosomal structure, quantity, centromere position and other morphological information) may be abnormal or mutated. Thus, it may generate a high possibility to cause diseases. Generally, the karyotype of chromosome G-bands is detected and analyzed using an optical microscope. However, it is difficult to detect the G-band structures for traditional optical microscopes on the nanometer scale. Herein, we have studied the detection method of chromosome G-band samples by atomic force microscopy (AFM) imaging. The structures of chromosome G-banding are studied with different trypsin treatment durations. The experiment result shows that the treatment duration of $20 \mathrm{~s}$ is the best time to form G-band structures. The AFM images show the structures of chromosome G-bands which cannot be observed under an optical microscope. This work provides a new way for the detection and diagnosis of chromosome diseases on the nanometer scale.
\end{abstract}

Keywords: Atomic force microscopy, chromosome, G-band, trypsin treatment

\section{Introduction}

The chromosome is a carrier of genetic information in a special winding manner, including DNA, RNA, histones and non-histones (Wolffe et al. 2000). The karyotype 
refers to all the chromosomes in the mitosis metaphase of a somatic cell, and the images are arranged in the order of their sizes and morphological characteristics. Chromosome karyotype analysis is an important method in cytogenetics, and contributes to the diagnosis and treatment of various clinical diseases, such as chromosomal diseases, leukemia and tumors (Panagopoulos et al. 2018; Peterson et al. 2019). G-banding is one of the most common banding technologies for karyotype analysis (Arora et al. 2019). After Giemsa staining, the histones combined with the loose fragments of DNA were stained as pale bands (G-negative bands), and the histones combined with the tight fragments of DNA were stained as dark bands (G-positive bands). Researchers analyzed that the morphological characterization of chromosome G-bands using optical microscopes and studied numerous G-band karyotype analysis methods based on optical microscopes (Cai et al. 2004; Howe et al. 2014). For instance, Zhang et al. (2014) reported that the optical microscope was used to detect chromosomal aberrations from the workers caused by ionizing radiation. However, the resolution of optical microscope was low and the details of chromosome aberrations were not able to be detected. Thus, it was difficult to provide accurate information about the structures of chromosome Gbands (Koleva et al. 2013; Yilmaz et al. 2018).

It was reported that the work on the chromosome imaging was done by AFM (Cai et al. 2018; Wu et al. 2006a,b). The structural nucleosomes were also studied using AFM (Stumme-Diers et al. 2019; Würtz et al. 2019; Pisano et al. 2019). In addition, AFM was used to cut specific fragments of the chromosome, extract the specific DNA information, and measure the chromosome volume (Fotiadis et al. 2002; Tsukamoto et al. 2006; McMaster et al. 1996; Yan et al. 2015; Yan et al. 2014; Di et al. 2011). As an imaging technology, AFM was used to obtain the chromosomal morphology and observe the aberrations, such as translocations, deletions and other features. Miranda et al. (2008) analyzed that the topography of the G-bands adsorbed on the glass in the early and mid-term human chromosomes by the amplitude modulation mode of AFM (AM-AFM). Researchers (Yang et al. 1995; Daban et al. 2011) have studied that the structures of chromatin fibers and individual nucleosomes could be clearly discerned with AFM. Qu et al. (2004) revealed that AFM was able to provide more information for the study of chromosome aberration induced by radiation, including visualizing the damage state of the chromosome at the hot points, as a basis of further research in the genomic level. In addition, the effect of trypsin on the structures of chromosome Gbanding was studied by AFM. Musio et al. (1997) showed that the trypsin treatment 
during G-banding caused changes in chromosome structures. Sahin et al. (2000) indicated that the prolonged treatment with trypsin resulted in a collapsed pattern of chromosomes. Wu et al. $(2006 a, b)$ revealed that contaminants were removed by the trypsin treatment on the chromosome surface. However, the chromosome structures were damaged.

In recent years, AFM has become an indispensable tool to detect the ultrastructure of chromosomes. It can provide more accurate information on the location of chromosome aberrations and detects the higher-order structures of chromosomes. Both mechanical and electrochemical properties of samples have been studied using AFM (Klausen et al. 2016; Xu et al. 2007). The image quality and resolution of the chromosome G-band structures obtained by AFM are better than those obtained by optical microscopes (Hoshiet al. 2001). However, the preparation method of chromosome G-band samples that is suitable for AFM imaging has rarely been reported.

In this work, the effects of drying time and trypsin treatment time on the G-banding structures of human chromosomes were studied by AFM. A sample preparation and detection method for G-banding chromosomes suitable for AFM imaging was proposed. The high-resolution imaging of G-band chromosomes by AFM can be potentially used for the accurate diagnosis and treatment of chromosomal diseases.

\section{Experimental}

\section{Sample preparation}

Peripheral blood lymphocytes were cultured at $37^{\circ} \mathrm{C}\left(5 \% \mathrm{CO}_{2}\right)$ in RPMI-1640 containing bovine serum albumin $(15 \%)$ for $72 \mathrm{~h}$, and then were used in the experiments when the cells underwent the logarithmic growth. Colchicine (Sangon Biotech, China; final concentration of $0.05 \mu \mathrm{g} / \mathrm{ml}$ ) was added to the culture, then mixed gently and incubated at $37^{\circ} \mathrm{C}$ for $1.5-2 \mathrm{~h}$ prior to the cells harvested. After the hypotonic treatment in the $0.075 \mathrm{mM} \mathrm{KCl}$ solution for $30 \mathrm{~min}$ at $37^{\circ} \mathrm{C}$, the chromosomes were fixed in the methanol acetic acid fixative with the 3:1 ratio (Li et al. 2015; Benn et al. 2008). The deposit was resuspended in 0.3-0.5 $\mathrm{ml}$ of fixative, and 1-2 drops of the cell suspension were dropped onto a glass slide from a height of $30-50 \mathrm{~cm}$. The tilt angle of glass slide is $6^{\circ}$ (for better spreading). Excess water on the slide was evaporated by the alcohol lamp. After air-dried for $24 \mathrm{~h}$, the glass slide was treated with $0.25 \%$ trypsin for 10,15 , 20, 25 and 30 s, respectively. After stained in the Giemsa solution (10\%) for $2.5 \mathrm{~min}$, the glass slide was rinsed gently with distilled water. 


\section{AFM measurements}

The prepared samples were imaged at room temperature using an atomic force microscope (Agilent 5500, USA) working in the contact mode. The AFM images were acquired with ContAl-G AFM probes (spring constant: $0.2 \mathrm{~N} / \mathrm{m}$ ). The curvature radius of the silicon nitride tip was about $10 \mathrm{~nm}$. The maximum scanning range was $99 \mu \mathrm{m}$. In every step of the image analysis process, the area of interest was primarily scanned with the image resolution of $512 \times 512$ pixels.

\section{Contact angle measurements}

The inclination angles of the cell suspension drop down to the glass slide were measured with a contact angle measuring system (Drop shape analyzer-DSA100, Germany) at room temperature. In the experiments, the tilt angle of the glass slide was $6^{\circ}$, and the dropping volume was set to $6 \mu \mathrm{L}$.

\section{Results and discussion}

The trypsinization changes the morphological features of chromosomes with the increased treatment time. In this study, the images of the G-banding process of chromosomes were obtained by AFM after the trypsin treatment. The AFM height images of chromosomes were detected without the trypsin treatment, as shown in Figure 1. Fig. 1a shows that the untreated chromosome surface structures are smooth and the sister chromatids appear spiral corresponding to each other. The experimental result is consistent with the previous work reported by other researchers (Ushiki et al. 2008). Fig. 1b shows that the high-definition 3D topography of the AFM and the details of chromosome surfaces are revealed. More chromosomal abnormalities such as small deletions, repetitions, inversions and translocations are found by AFM, which can make the location of chromosome aberrations more precise and improve the accuracy of diagnosis (Koleva et al. 2013). 

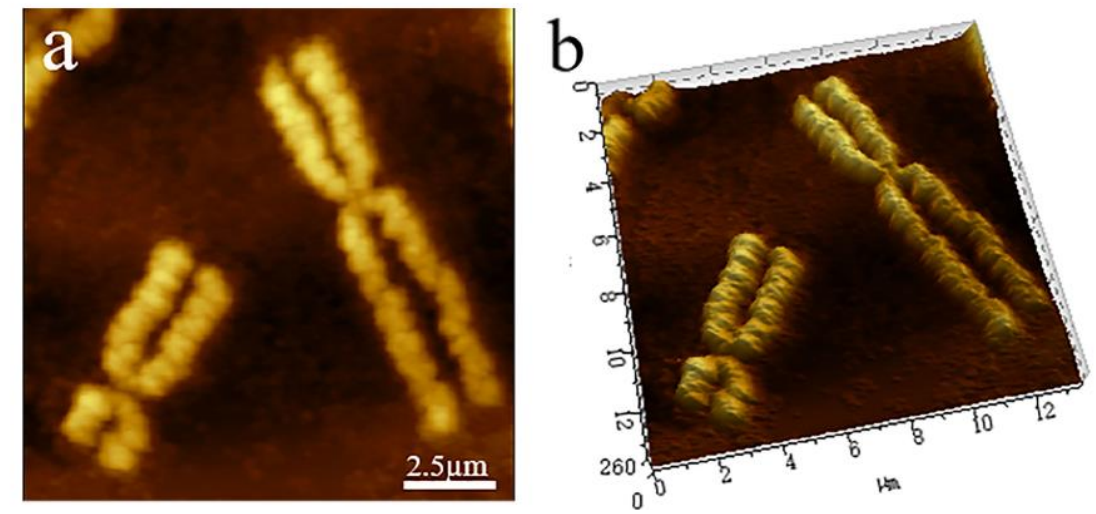

Fig. 1 AFM height images of chromosomes without the trypsin treatment (a). Three-dimensional images of chromosomes (b)

Furthermore, the chromosome G-banding structures were studied with different time durations of trypsinization at the same concentration, as shown in Figure 2. The chromosomes treated with trypsin for10, 15, 25 and $30 \mathrm{~s}$ were imaged and analyzed by AFM. Figs. 2a-2h show that the effect of trypsin duration on chromosome structures can be directly observed from AFM height and three-dimensional images, respectively. When the trypsin treatment time was $10 \mathrm{~s}$, the regular spiral structures become smooth and the bands are not generated, as shown in Figures 2a and 2e. When the trypsin treatment time was $15 \mathrm{~s}$, a very shallow band structure appeared on the surface of the chromosome, as shown in Figures $2 b$ and $2 f$. However, the structure was irregular. Figs. $2 \mathrm{c}$ and $2 \mathrm{~g}$ show that the structure of chromosomes is collapsed with the trypsin treatment for $25 \mathrm{~s}$ and the internal differentiation between chromatids disappears, and there is a structure similar to a ladder-shaped pit. In addition, the subsequent structures of the chromosome were generated with the trypsin for $30 \mathrm{~s}$, as shown in Figures $2 \mathrm{~d}$ and $2 \mathrm{~h}$. Almost all internal structures except the chromosome edges were treated by trypsin. Figs. 2i-2l are the height profiles of the transversal sections marked with the white dashed lines in the height images of (a)-(d). The profiles indicated that the prolonged treatment with trypsin resulted in the decrease of chromosome height, and the increase of surface roughness and irregularity. Therefore, appropriate treatment time will make the surface structures of chromosomes regularly. If the treatment time is too short, the surface of the chromosome can be treated only a little and the G-bands cannot be clearly analyzed. With the extension of treatment time, the internal structures of the chromosome were gradually dissolved, and the edges of the chromosome were obvious. 

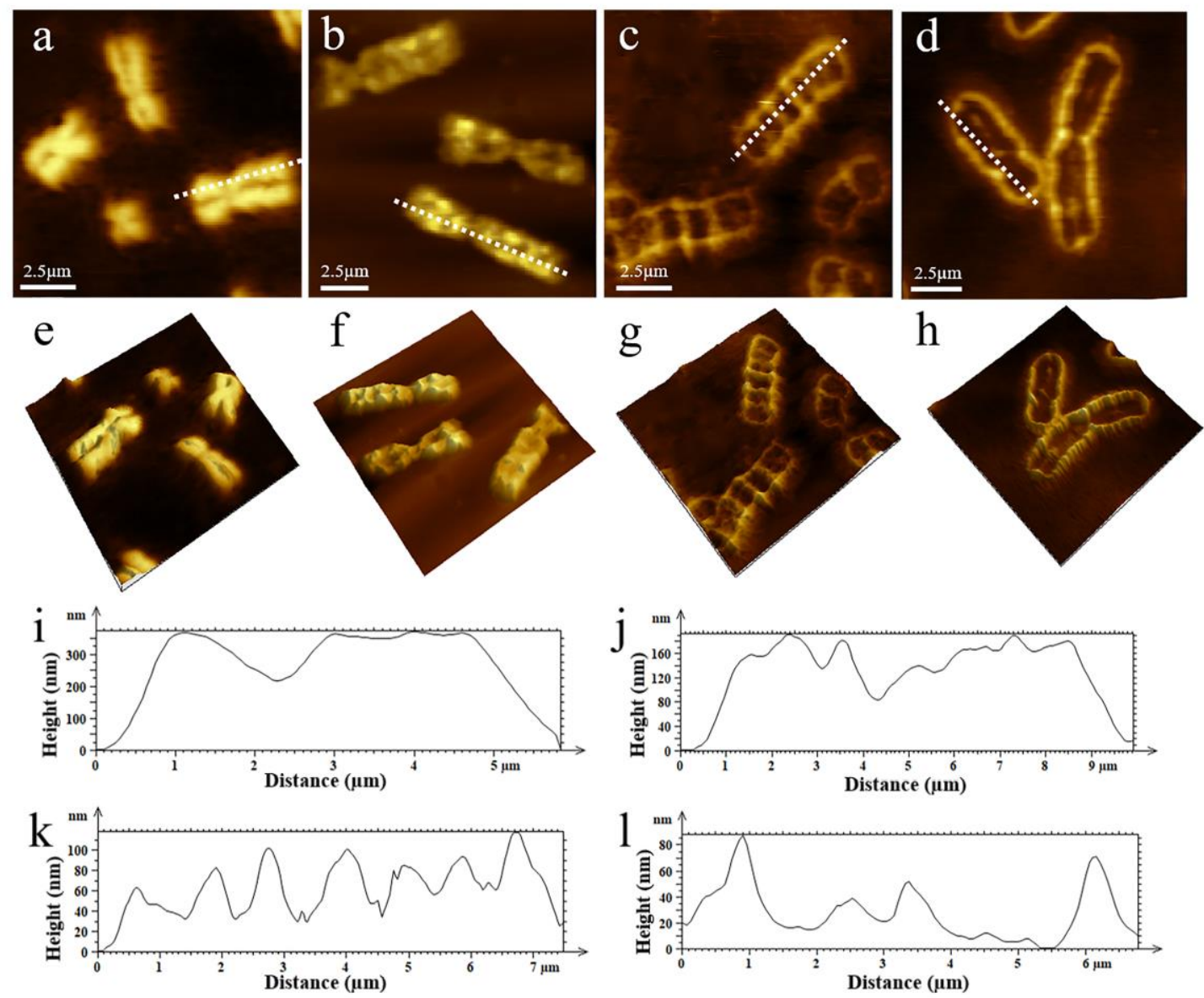

Fig. 2 AFM height and three-dimensional images of chromosome structural changes caused by trypsin treatment: (a and e) after $10 \mathrm{~s}$, (b and f) after $15 \mathrm{~s}$, (c and g) after $25 \mathrm{~s}$, and (d and h) after 30 s. (i)-(l) are the height profiles of the transversal sections marked with the white dashed lines in the height images of (a)-(d)

Based on the effect of trypsin treatment time on the structures of chromosome Gbanding, an experimental method suitable for the AFM imaging of G-bands is presented. Figs. $3 \mathrm{a}$ and $3 \mathrm{~b}$ show that the structures of chromosome G-bands are treated by trypsin for $20 \mathrm{~s}$ imaged with the AFM and optical microscope. In order to ensure the bands to correspond, the white arrows and the black arrows are the corresponding bands, respectively, as shown in Figures $3 \mathrm{a}$ and $3 \mathrm{~b}$. It can be seen from Figure $3 \mathrm{a}$ that the images of chromosome G-bands obtained with AFM are clear and the details are visible. Fig. $3 b$ shows the images of chromosome G-bands obtained with the optical microscope are blurred. Furthermore, the G-positive bands of chromosomes are significantly higher than the G-negative bands obtained from AFM images. In contrast, the pale (G-negative) and dark (G-positive) stripes of chromosomes obtained by the optical microscope are 
not obvious. With the low resolution of optical microscope, there is a possibility of missing the important details. Just like the negative bands at the ends of chromosomes circled by dashed lines, they are shown in Figures $3 \mathrm{a}$ and $3 \mathrm{~b}$. In addition, the G-bands of chromosomes and their height curves are obtained directly in the 3D AFM images. The positions of the positive bands of the chromosome correspond to the "peaks" of cross-sectional AFM images. In addition, the positions of the negative bands of the chromosome correspond to the "troughs" of cross-sectional AFM images, as shown in Figures $3 \mathrm{c}$ and $3 \mathrm{~d}$. That is, the "peaks" and "troughs" of chromosomes correspond to the positive and negative bands of the pattern diagram, respectively. It is demonstrated that the AFM images of the chromosome structures can reflect the unique banding pattern of each chromosome.
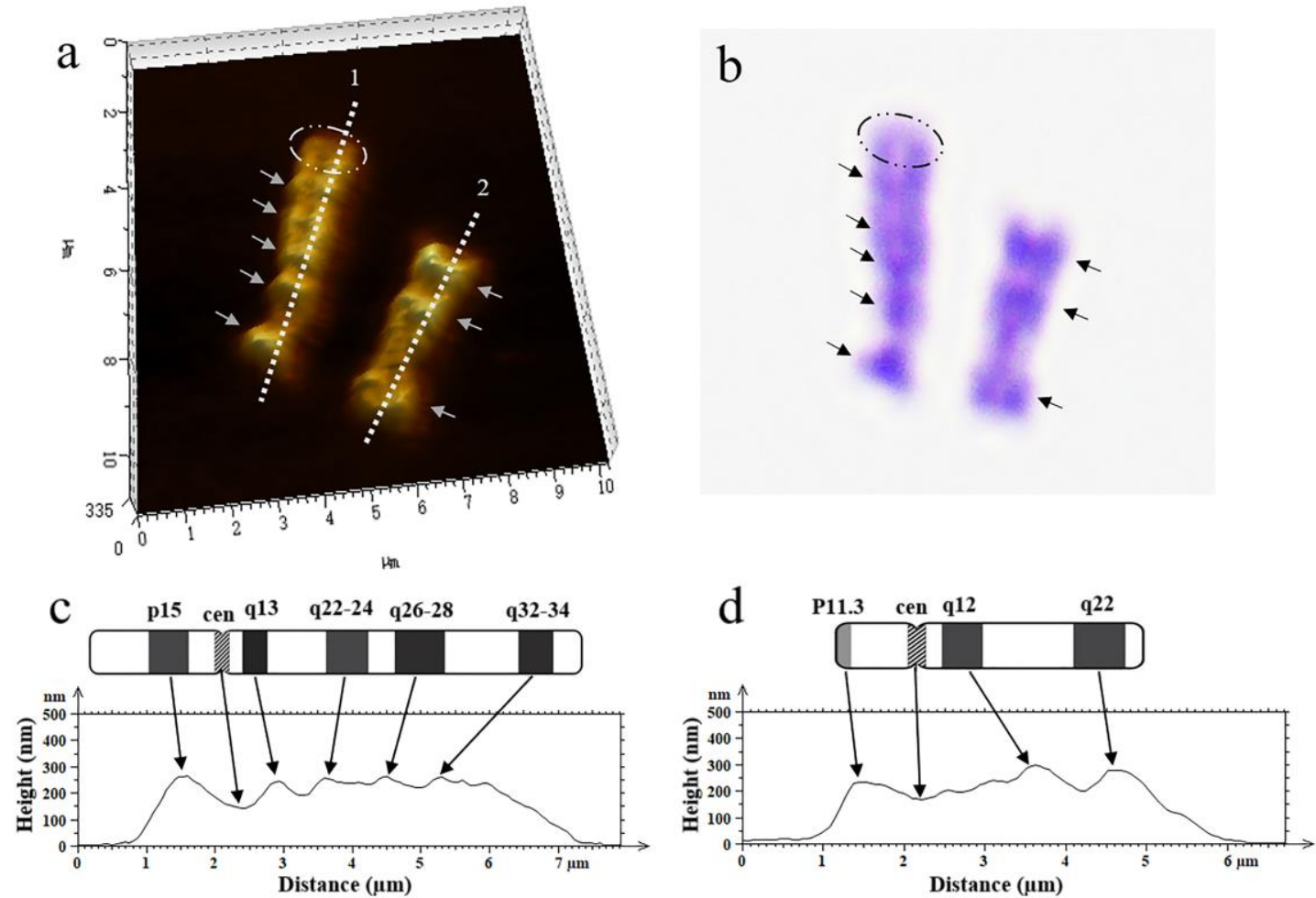

Fig. 3 Images of chromosome G-bands treated by trypsin for 20 s: (a) Three-dimensional AFM images of chromosomes. (b) Optical microscope images of chromosomes. (Note: The white arrows and the black arrows are the corresponding bands.) (c) The above is the pattern diagram of chromosome 4. From left to right, the names of the positive bands of some short arms, centromeres, and some long arms are marked in order. Below is the vertical height variation curve of chromosome 4 (selected from dotted line 1 of chromosome 4 in Fig. 3a). The peaks of the curve indicated by the black arrows show the locations of the chromosome positive bands. (d) The above is the pattern 
diagram of chromosome 18. From left to right, the names of the positive bands of some short arms, centromeres, and some long arms are marked in order. Below is the longitudinal height variation curve of chromosome 18 (selected from dotted line 2 of chromosome 18 in Fig. 3a). The peaks of the curve indicated by the black arrows show the locations of the chromosome positive bands

In this study, the chromosomes have a better dispersion, when the cell suspension drops down to the glass slide tilted at a certain angle. According to the results of contact angle measurement and optical microscope observation as shown in Figure 4, when the inclination angle of the glass slide is $6^{\circ}$, the cell suspension can spread well without causing the cell suspension to slip off due to the excessive inclination angle. Thus, the method adopted in this study will make the chromosome structures non-overlapped and the chromosome sample suitable for AFM imaging and analysis.
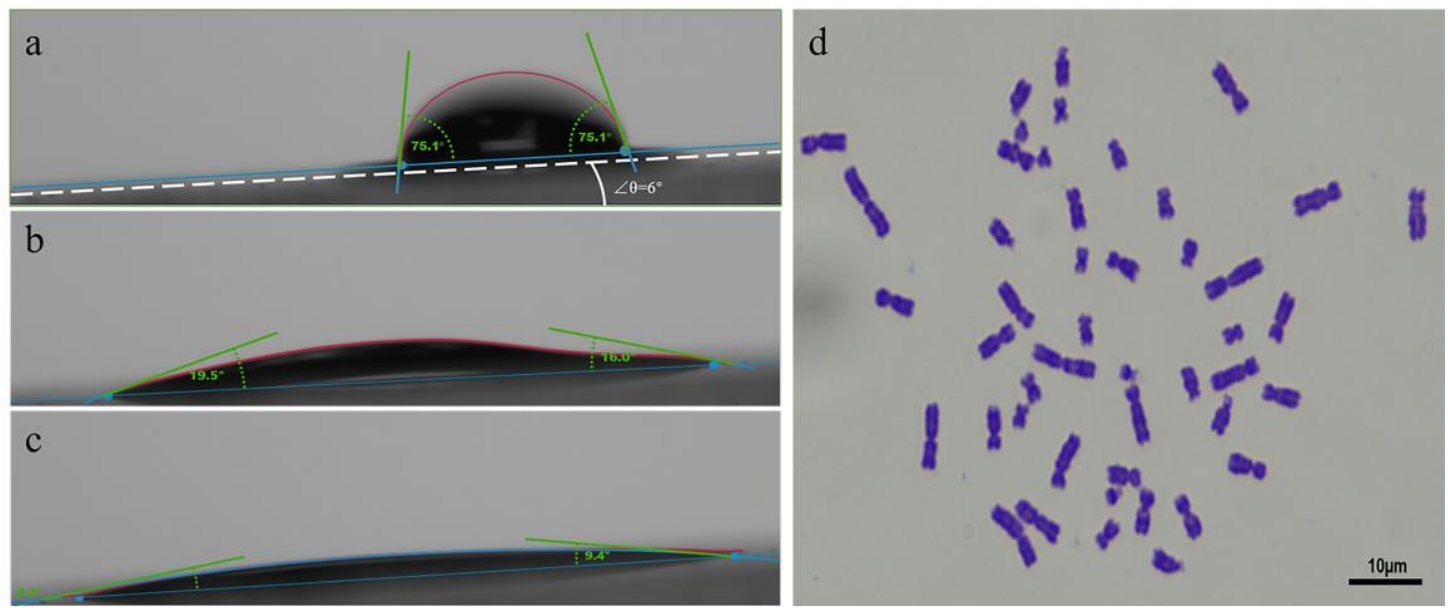

Fig. 4 Contact angle images of cell suspension and the optical image of chromosomes with good dispersion: the cell suspension in contact with the glass slide (a), the cell suspension is tilted on the glass slide (b), the cell suspension extends down the slope (c), and the chromosomes with good dispersion on the glass slide (d). (Note: The tilt angle of the glass slide is $6^{\circ}$.)

Besides, Fig. 5 shows that the AFM images of four groups of chromosomes are treated with trypsin for $20 \mathrm{~s}$ with different dry time durations. Fig. 5a shows the treatment with trypsin directly without a drying process after the preparation of the chromosome sample. The band structures disappear and are replaced by the fibrous structures. When the drying time is $12 \mathrm{~h}$, the chromosome structures are also destroyed at the same treatment time, and some bands are visible, as shown in Fig. 5b. The chromosome fibers resulted from insufficient aging time can affect the observation of 
G-banding. When the drying time is $24 \mathrm{~h}$, the chromosome surface is smooth and the bands are clear, as shown in Fig. 5c. However, when the drying time is extended to 48 h, only few structures are treated on the surfaces of the chromosomes, as shown in Fig. 5d. The periodicity of surface structures can be analyzed using FFT (Liang al. 2016). Figs. 5e-5h show that the periodicities of chromosome G-bands are reflected by the bright lines passing through the center points of Fourier transform images. Fig. $5 \mathrm{~g}$ indicates that the bright lines in the Fourier transform image confirm the clear bands of chromosomes in Figure 5c. Because the prolonged drying time will make the chromosomes aging, the histones of the chromosome are not easily treated by trypsin, and influence the form of band structures. It can be seen that the chromosome samples with different drying time durations have different conditions for the treated G-band structures.
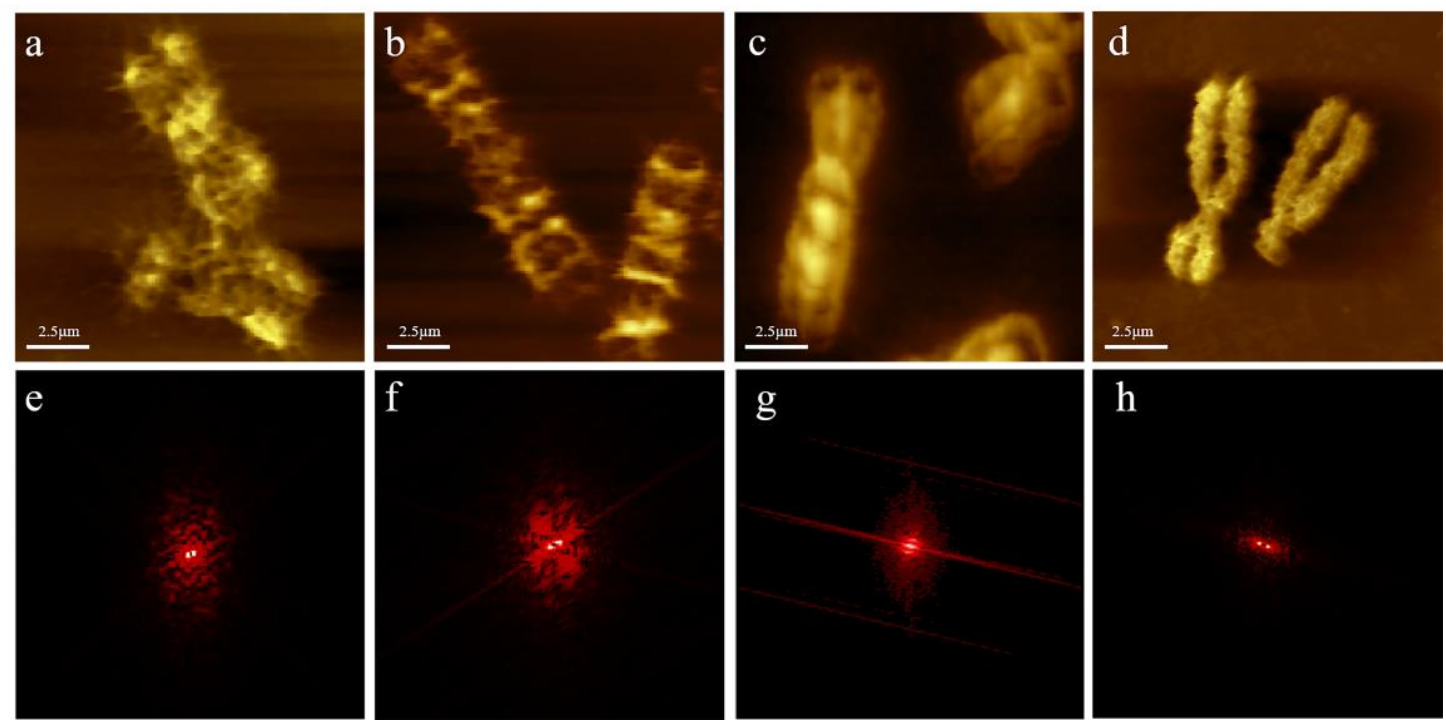

Fig. 5 AFM images of samples with different drying time durations of $0 \mathrm{~h} \mathrm{(a),} 12 \mathrm{~h}$ (b), $24 \mathrm{~h}$ (c) and $48 \mathrm{~h}$ (d), and treated with trypsin for $20 \mathrm{~s}$, respectively. (e)-(h) are corresponding Fourier transform images of the AFM images (a)-(d)

\section{Conclusion}

In summary, the AFM images of chromosome G-bands were successfully observed. The chromosome samples were obtained with the drying time of $24 \mathrm{~h}$ and the trypsin treatment time of $20 \mathrm{~s}$, and the chromosome samples of G-band structures were suitable for AFM imaging. The samples prepared by this method can not only fully evaporate the fixing solution, but also make the samples aging moderately. The sample treatment time with trypsin is easy to be managed. The AFM image has clear G-band 
structures and can achieve the best banding result. The images of chromosome G-bands obtained by AFM correspond to the bands from the optical microscope imaging. AFM has a higher resolution to detect more bands than optical microscopy. Besides, the AFM image is more accurate in the position of G-bands. Therefore, the G-bands of chromosome samples are suitable for AFM imaging. Observing structural changes can provide more accurate information of chromosomal aberrations on the nanometer scale, and it is important for the chromosome karyotype analysis and the diagnosis of chromosomal diseases. The AFM images of the chromosome G-band structures are expected to replace the conventional karyotype analysis methods using optical microscopes or be used in conjunction with them to provide a new way for the detection and diagnosis of chromosomes.

\section{Acknowledgements}

This work was supported by National Key R\&D Program of China (No. 172017YFE0112100), EU H2020 Program (MNR4SCell No.734174), Jilin Provincial Science and Technology Program (Nos.20180414002GH, 20180414081GH, 20180520203JH, 20190702002GH and 20200901011SF), and "111" Project of China (D17017).

\section{Conflict of interest}

The authors declare that they have no conflict of interest.

\section{References}

Wolffe A P, Guschin D (2000) Chromatin structural features and targets that regulate transcription. Journal of Structural Biology 129(2-3): 102-122

Panagopoulos I, Gorunova L, et al (2018) RUNX1-PDCD6 fusion resulting from a novel $\mathrm{t}(5 ; 21)(\mathrm{p} 15 ; \mathrm{q} 22)$ chromosome translocation in myelodysplastic syndrome secondary to chronic lymphocytic leukemia. Plos One 13(4): e0196181

Peterson J F, Pitel B A, Smoley S A, et al (2019) Constitutional chromosome rearrangements that mimic the 2017 world health organization "acute myeloid leukemia with recurrent genetic abnormalities": A study of three cases and review of the literature. Cancer Genetics 230: 3746

Arora T, Dhir R (2019) A novel approach for segmentation of human metaphase chromosome images using region based active contours. Int. Arab J. Inf. Technol 16(1): 132-137

Cai N, Hu K, Xiong H, et al (2004) Image segmentation of $\mathrm{G}$ bands of triticum monococcum chromosomes based on the model-based neural network. Pattern Recognition Letters 25(3): 319-329

Howe B, Umrigar A, Tsien F (2014) Chromosome preparation from cultured cells. Journal of Visualized Experiments (83): e50203 
Zhang X, Zhang Y, Zhong G (2014) Influence of ionizing radiation on micronucleus, chromosome aberration and leukocyte among radiation workers. Occup Health 13:1724Koleva V P, Dragoeva A P, Andreeva A I, et al (2013) Comparative analysis of clastogen-induced chromosome aberrations observed with light microscopy and by means of atomic force microscopy. Mutation Research/Genetic Toxicology and Environmental Mutagenesis 753(1): 29-35

Yilmaz I C, Yang J, Altinsoy E, et al (2018) An improved segmentation for raw G-band chromosome images. IEEE 2018 5th International Conference on Systems and Informatics 944-950

Cai H H, Zeng X, Tang X, et al (2018) Atomic force microscopy: a nanoscopic application in molecular and cell biology. At Force Microsc Mol Cell Biol (Chapter 5):77-103Wu Y, Cai J, Cheng L, et al (2006a) Chromosome imaging by atomic force microscopy: influencing factors and comparative evaluation. Journal of Genetics 85(2): 141-145

Stumme-Diers M P, Stormberg T, Sun Z, et al (2019) Probing the structure and dynamics of nucleosomes using atomic force microscopy imaging. Journal of Visualized Experiments (143): e58820

Würtz M, Aumiller D, Gundelwein L, et al (2019) DNA accessibility of chromatosomes quantified by automated image analysis of AFM data. Scientific Reports 9(1): 1-12

Pisano S, Gilson E (2019) Analysis of DNA-protein complexes by atomic force microscopy imaging: the case of TRF2-telomeric DNA wrapping. Atomic Force Microscopy. Humana Press, New York, pp 75-97

Fotiadis D, Scheuring S, Müller S A, et al (2002) Imaging and manipulation of biological structures with the AFM. Micron 33(4): 385-397

Tsukamoto K, Kuwazaki S, Yamamoto K, et al (2006) Dissection and high-yield recovery of nanometre-scale chromosome fragments using an atomic-force microscope. Nanotechnology 17(5): 1391-1396

McMaster T J, Winfield M O, Baker A A, et al (1996) Chromosome classification by atomic force microscopy volume measurement. Journal of Vacuum Science \& Technology B: Microelectronics \& Nanometer Structures Processing, Measurement\& Phenomena 14(2): 1438-1443

Yan Y, Geng Y, Hu Z (2015) Recent advances in AFM tip-based nanomechanical machining. International Journal of Machine Tools \& Manufacture Design Research \& Application 99: 118

Yan Y, Geng Y, Hu Z, et al (2014) Fabrication of nanochannels with ladder nanostructure at the bottom using AFM nanoscratching method. Nanoscale Research Letters 9(1): 212

Di Bucchianico S, Poma A M, Giardi M F, et al (2011) Atomic force microscope nanolithography on chromosomes to generate single-cell genetic probes. Journal of Nanobiotechnology 9(1): 17

Argüello - Miranda O, Sáenz - Arce G (2008) Interchromatidal central ridge and transversal symmetry in early metaphasic human chromosome one. Journal of Molecular Recognition: An Interdisciplinary Journal 21(3): 184-189

Yang G, Leuba S S, Bustamante C J, et al (1995) Scanning force microscopy study of native and linker histone-depleted chromatin fibers. Scanning Probe Microscopies III. International Society for Optics and Photonics 2384: 13-21

Daban J R (2011) Electron microscopy and atomic force microscopy studies of chromatin and 
metaphase chromosome structure. Micron 42(8): 733-750

Qu S, Chen Y, Ge S, et al (2004) Study of chromosome translocation in victims of a previous radiation accident by atomic force microscopy. Chinese Journal of Radiological Medicine and Protection 24(4): 353-356

Musio A, Sbrana I, Mariani T, et al (1997) Atomic force microscope imaging of chromosome structure during G-banding treatments. Genome 40(1): 127-131

Şahin F I, Ergün M A, Tan E, et al (2000) The mechanism of G-banding detected by atomic force microscopy. Scanning 22(1): 24-27

Wu Y, Cai J, Cheng L, et al (2006b) Atomic force microscopic examination of chromosomes treated with trypsin or ethidium bromide. Chemical and Pharmaceutical Bulletin 54(4): 501-505

Klausen L H, Fuhs T, Dong M (2016) Mapping surface charge density of lipid bilayers by quantitative surface conductivity microscopy. Nature communications 7(1): 1-10

Xu S, Dong M, Liu X, et al (2007) Direct force measurements between siRNA and chitosan molecules using force spectroscopy. Biophysical journal 93(3): 952-959

HOSHI O, Ushiki T (2001) Three-dimensional structure of G-banded human metaphase chromosomes observed by atomic force microscopy. Archives of Histology and Cytology 64(5): $475-482$

Li F, Ma L, Liu B, et al (2015) A new method of extracting the altitude curves along chromosomes based on contour line. International Conference on Manipulation. IEEE 98-102

Benn P, Delach J (2008) Human lymphocyte culture and chromosome analysis. Cold Spring Harbor Protocols 2008(10): pdb. prot5035

Ushiki T, Shigeno M, Hoshi O (2008) Techniques for imaging human metaphase chromosomes in liquid conditions by atomic force microscopy. Nanotechnology 19(38): 384022

Liang $\mathrm{C}$, Hu Y, Wang $\mathrm{H}$, et al (2016). Biomimetic cardiovascular stents for in vivo reendothelialization. Biomaterials 103: 170-182 